\title{
Molecular MRI of Inflammation in Atherosclerosis
}

\author{
Bernard C. te Boekhorst • Geralda A. van Tilborg • \\ Gustav J. Strijkers • Klaas Nicolay
}

Published online: 5 November 2011

(C) The Author(s) 2011. This article is published with open access at Springerlink.com

\begin{abstract}
Inflammatory activity in atherosclerotic plaque is a risk factor for plaque rupture and atherothrombosis and may direct interventional therapy. Inflammatory activity can be evaluated at the (sub)cellular level using in vivo molecular MRI. This paper reviews recent progress in contrast-enhanced molecular MRI to visualize atherosclerotic plaque inflammation. Various MRI contrast agents, among others ultra-small particles of iron oxide, lowmolecular-weight Gd-chelates, micelles, liposomes, and perfluorocarbon emulsions, have been used for in vivo visualization of various inflammation-related targets, such as macrophages, oxidized LDL, endothelial cell expression, plaque neovasculature, MMPs, apoptosis, and activated platelets/thrombus. An enzyme-activatable magnetic resonance contrast agent has been developed to study myeloperoxidase activity in inflamed plaques. Agents creating contrast based on the chemical exchange saturation transfer mechanism were used for thrombus imaging. Transfer of
\end{abstract}

\author{
B. C. te Boekhorst $(\bowtie) \cdot$ G. J. Strijkers $\cdot$ K. Nicolay \\ Biomedical NMR, Department of Biomedical Engineering, \\ Eindhoven University of Technology, \\ Den Dolech 2, \\ 5612 AZ Eindhoven, The Netherlands \\ e-mail: b.t.boekhorst@tue.nl \\ G. J. Strijkers \\ e-mail: g.j.strijkers@tue.nl \\ K. Nicolay \\ e-mail: k.nicolay@tue.nl \\ G. A. van Tilborg \\ Biomedical MR Imaging and Spectroscopy Group, \\ Image Sciences Institute, University Medical Center Utrecht, \\ Yalelaan 2, \\ 3584 CM Utrecht, The Netherlands \\ e-mail: gtilborg@gmail.com
}

these molecular MRI techniques to the clinic will critically depend on the safety profiles of these newly developed magnetic resonance contrast agents.

Keywords Molecular MRI · Cellular MRI · Inflammation · Atherosclerosis · Iron oxide - Gadolinium · Nanoparticles . USPIO $\cdot$ Micelle $\cdot$ Liposome $\cdot$ Perfluorocarbon nanoparticle $\cdot$ HDL-like nanoparticle $\cdot$ Activatable contrast agent $\cdot$ Macrophages $\cdot$ Ox-LDL $\cdot$ Vascular adhesion molecules $\cdot$ MMPs $\cdot$ Myeloperoxidase $\cdot$ Integrins $\cdot$ Activated platelets $\cdot$ Apoptosis $\cdot$ Fibrin

\section{Introduction}

Atherothrombotic diseases contribute to a major portion of mortality in the Western world. Inflammatory activity in the atherosclerotic plaque is a key player in the progress of atherosclerosis and the risk for plaque rupture [1]. Sensitive and specific imaging biomarkers are highly desired for prognostication of atherosclerosis in the high-risk population. Local plaque imaging biomarkers, which can direct interventional cardiologists and vascular surgeons to diseased sites, are therefore of great interest. At present, serologic markers do not accomplish this need sufficiently.

Inflammatory activity can be evaluated at the cellular (macrophages, neutrophils, and lymphocytes) or subcellular (secreted and intracellular enzymes, membrane-bound proteins) level. Various noninvasive imaging techniques are available for evaluation of the levels of these inflammationrelated cells, proteins, or enzymes. PET, SPECT, CT, ultrasound, and MRI are all clinically available imaging modalities that could play a role in molecular imaging of plaque inflammation [2]. MRI has the distinct advantage that it is safe, does not involve ionizing radiation, has a high 
penetration depth, and is capable of providing molecular information with high spatial resolution in an anatomical and physiological context.

During the process of atherogenesis, inflammation aggravates endothelial dysfunction and vice versa. Various more or less sequential processes take place during the development from a fatty streak to a rupture-prone or ruptured plaque [1]. First, low-density lipoprotein (LDL) uptake into the sub-intima is followed by oxidation of LDL by tyrosyl radicals and aldehydes, which result from myeloperoxidase produced by plaque neutrophils and macrophages. Oxidized LDL (ox-LDL) stimulates endothelial cell dysfunction, leading to inflammatory cell migration and activation. Recruited macrophages release matrixdegrading enzymes and other factors that eventually result in apoptosis of smooth muscle cells, endothelial cells, and macrophages. Eventually, these processes may lead to fibrous cap thinning, lipid core growth, formation of fragile intra-plaque neovasculature, intra-plaque hemorrhage, and finally fibrous cap rupture. Subsequent formation of a thrombus may cause an acute thrombotic event.

Why some atherosclerotic plaques remain stable and why certain plaques develop into rupture-prone plaques at a fast pace, is currently unknown and therefore the subject of intense research. It is hypothesized that the inflammatory status of the plaque plays an important role in the development of so-called unstable plaque. In this review, we will therefore focus on recent progress in molecular MRI using contrast agents to noninvasively visualize atherosclerotic plaque inflammation. In the following sections we will discuss a number of targets, according to the sequence of atherosclerotic processes (ox-LDL, endothelial cells, neovascularization, macrophages, apoptotic cells, activated platelets, thrombus), that have been used to image various aspects of the inflammatory pathways in atherosclerosis.

\section{Ox-LDL}

Targeting specific epitopes of ox-LDL in atherosclerotic plaque has successfully been demonstrated using antibody-conjugated paramagnetic micelles. Application of ox-LDL-targeted micelles in apoE-/- mice resulted in a specific MRI signal enhancement of plaques in the aortic wall at 72 and $96 \mathrm{~h}$ after intravenous injection in comparison to the use of control non-conjugated micelles and nonspecific IgG-conjugated micelles [3]. Despite this success, clinical application of targeted micelles may be limited due to the observed long blood circulation times $(>14 \mathrm{~h})$ and high liver uptake $(20 \%$ of the injected dose). Although the use of gadolinium $(\mathrm{Gd})$ is generally believed to be safe, excessive retention of Gd- chelates in organs needs to be avoided, as Gd toxicity was recently implicated in the occurrence of cell apoptosis $[4$, 5] and nephrogenic systemic fibrosis (NSF) in patients with renal dysfunction [6].

Briley-Saebo and coworkers [7] also developed a lipidcoated ultra-small superparamagnetic iron particles (LUSPIOs) $(<20 \mathrm{~nm})$ targeted to oxidation-specific epitopes on ox-LDL in the plaque of apoE-/- mice. Upon in vivo application in apoE- $/-$ mice, enhancement of the aortic wall was observed in gradient echo images, using a positive contrast method (GRASP), whereas no enhancement was observed for control nontargeted LUSPIOs and larger $(\sim 40 \mathrm{~nm})$ LSPIOs. Coating the particles with lipids prevented nonspecific uptake by macrophages, leading to higher in vivo specificity for ox-LDL.

\section{Endothelial Cells}

Inflammation leads to increased expression of cell adhesion molecules like ICAM-1, VCAM-1, and P-selectin by endothelial cells via the inflammatory mediators IL-1 and TNF- $\alpha$. VCAM-1 expression is also induced by oxidized low-density lipoproteins, which accumulate in the plaque during atherogenesis. VCAM-1 is bound by very late antigen-4 (VLA-4) on the cell surface of leukocytes mediating their extravasation. McAteer et al. [8] designed microparticles (diameter $\sim 4.5 \mu \mathrm{m}$ ) of iron oxide conjugated with antibodies directed against VCAM-1. In vitro, the binding capacity of these microparticles to TNF- $\alpha$-stimulated mouse endothelial cells was demonstrated [8]. In vivo, the VCAM-1-targeted microparticles were used to image inflammatory changes to peritubular capillaries in murine kidneys after ischemia reperfusion injury [9]. A crosslinked iron oxide (CLIO) nanoparticle was applied to target VCAM-1 expression by dysfunctional aortic endothelium of cholesterol-fed apoE-/- mice $[10,11]$. The CLIOs were also equipped with a fluorescent moiety for fluorescence imaging and VCAM-1 targeting was achieved by conjugation of a peptide sequence with homology to the a-chain of VLA-4.

As a response to the upregulation of inflammatory mediators, endothelial cells express P-selectin, which interacts with P-selectin glycoprotein ligand-1. This interaction involves some basic amino acids of P-selectin and sulfate residues carried by PSGL-1. The latter interaction was exploited to target P-selectin in plaques of apoE-/mice with a slow-clearing blood-pool agent functionalized with sulfate groups [12]. Application of this P-selectintargeted agent led to specific plaque enhancement between 10 and $30 \mathrm{~min}$ after intravenous injection. Ex vivo fluorescence microscopy confirmed specific binding of the agent to P-selectin. 


\section{Neovascularization}

The formation of neoangiogenic vessels has been associated with the hypoxic conditions in the thickened intimal layer $(>500 \mu \mathrm{m})$ [13]. Recent observations, however, suggest that neovascularization may promote plaque inflammation and progression already at an early stage of plaque formation by facilitating extravasation of inflammatory cells [14]. Rapid carotid atherosclerotic plaque growth has also been related to intraplaque hemorrhage of the fragile neovasculature [15].

Dynamic contrast enhancement (DCE) MRI, a technique well known for its ability to evaluate tumor vascularity, recently finds new application in the assessment of human plaque neovascularization [16]. DCE-MRI uses serial MRI acquisitions with high temporal resolution before and following the injection of a low-molecular-weight Gdcontaining contrast agent. DCE-MRI has demonstrated the ability to differentiate fibrous tissue (enhancement $\sim 80 \%$ ) from lipid core (enhancement $\sim 30 \%$ ) by virtue of the neovessel density in fibrous tissue [16]. Appropriate modeling of the signal intensity time course in the plaque provides information on contrast agent plaque uptake kinetics and estimations of the amount of neovascularization and vessel permeability [17].

The above-described targeted approaches with Gd-based contrast agents require high payloads of Gd accumulating at the site of interest to generate sufficient contrast in the MR images. Gadofluorine M (Bayer Schering Pharma AG) is an amphiphile with perfluorinated side chains, which forms micelles with a diameter of 5 to $6 \mathrm{~nm}$ in watery solution. Gadofluorine $\mathrm{M}$ micelles accumulate in high concentrations in atherosclerotic plaque, delivering an amount of $\mathrm{Gd}$ which results in a high contrast-to-background ratio in $\mathrm{T}_{1^{-}}$ weighted MRI [18]. Accumulation of micelles in the plaque was attributed to enhanced vessel-wall permeability and affinity of Gadofluorine $M$ for plaque lipids [18]. Additionally, affinity of Gadofluorine $\mathrm{M}$ for extracellular matrix proteins was observed [19]. Recently, Gadofluorine M uptake in the balloon-injured aorta of New Zealand white rabbits has been found to correlate with plaque neovessel and macrophage density [20]. Histological evaluation showed that plaque neovessels, lipid-rich regions, and Gadofluorine M co-localized.

Also targeted approaches were used for the molecular MR imaging of plaque neovascularization. Targeting of endothelial receptors indicative for neovascularization has the distinct advantage that the contrast agent need not enter the plaque itself, which allows for the use of large, very potent, nanoparticulate MR contrast agents. For example, Gd-containing perfluorocarbon emulsion nanoparticles with a size of about $250 \mathrm{~nm}$ were used to target plaque neovascularization [21]. It was demonstrated that an increased vasa vasorum density characterizes advanced atherosclerotic plaque.

The rat with a mutation in the leptin receptor is a model for metabolic syndrome, resulting in abdominal obesity, atherosclerosis, and myocardial lesions. Neovascularization in plaques in the aorta of these rats was imaged using a Gdcontaining perfluorocarbon emulsion targeted toward the $\alpha_{v} \beta_{3}$ integrin expressed on the vascular endothelium [21]. Additionally, the influence on plaque neovascularization of treatment with the anorectic and hypolipidemic drug benfluorex was investigated. Treated animals displayed an overall lower and patchy plaque enhancement in comparison to non-treated ones, indicative for a decreased angiogenic vessel density in the plaque. This study demonstrated successful application of these molecular MRI approaches to study neovascularization in atherosclerosis and changes therein upon treatment. The same $\alpha_{\mathrm{v}} \beta_{3^{-}}$ targeted nanoparticles were used to study treatment with angiogenic L-arginine in a rabbit femoral artery ligation model [22]. The achieved results in these diverse studies warn of one-sided interpretation of MR imaging results in atherosclerosis using $\alpha_{v} \beta_{3}$-targeted nanoparticles. Molecular MRI of neovascularization with these nanoparticles may not discriminate between adverse plaque neovessels and beneficial sprouting of collaterals.

\section{Macrophages}

Using molecular MRI, probably the most extensively studied plaque components are the macrophages. Previously, dextrancoated USPIOs (diameter 18-30 nm) were applied to discriminate healthy lymph nodes from ones that contain metastatic tumor tissue [23]. One of these MRI studies showed upon careful analysis not only ingestion of these USPIOs by macrophages in the lymph node but also by aortic plaque macrophages [24]. Nevertheless, accumulation of iron oxides in lymph nodes may complicate their application to detect macrophages in atherosclerotic plaque, since lymph nodes often develop in close proximity to the inflamed plaque lesion. We have shown that differentiation between perivascular lymph nodes and aortic wall in atherosclerotic mouse models may be difficult using USPIOs (diameter 18-30 $\mathrm{nm}$ ) and successful visualization will depend on resolution and the imaging sequence (fast spin echo instead of gradient echo) [25]. Recently, it was shown that the GRASP imaging technique, producing positive contrast with SPIOs, was capable of differentiating the perivascular lymph nodes from the vessel wall [7].

USPIOs have also found their way to the clinic for atherosclerotic plaque imaging. In a study by Kooi et al. [26], patients who were scheduled for carotid endarterectomy were first scanned by MRI and next injected with USPIOs. At 
$24 \mathrm{~h}$ after injection, $\mathrm{T} 2 *$-weighted images of the carotid artery wall showed decreased signal intensity, which normalized at $72 \mathrm{~h}$ after injection [26]. Following surgical removal of the lesioned carotid artery, $75 \%$ of ruptured and rupture-prone plaques were positive for USPIOs on histological analysis compared to $7 \%$ of stable plaques [26]. Investigation of the contralateral side in patients with symptomatic carotid stenosis demonstrated uptake of USPIOs in $95 \%$ of plaques, despite a mean stenosis of only $46 \%$ [27]. This result emphasizes the truly systemic nature of atherosclerosis. Also the difference in effect between aggressive and mild lipidlowering therapy on atherosclerotic plaque macrophages of patients was successfully investigated, using USPIO-based detection by MRI [28].

Specific targeting of macrophages in murine plaque has been achieved with paramagnetic Gd-containing micelles directed toward the membrane-bound macrophage scavenger receptor-A (MSR-A). Plaques in the aortic wall of apoE-/mice displayed a $79 \%$ increase in signal intensity upon injection of the MSR-A-targeted micelles, whereas control micelles resulted in 34\% signal increase [29]. MSR-A together with MSR-B is responsible for $75 \%-90 \%$ of oxLDL uptake by macrophages. The latter has been targeted with 125-nm-diameter lipid-based nanoparticles ex vivo in dissected human aortas containing atherosclerotic lesions [30]. However, in vivo application of MSR-B-targeted nanoparticles has not been demonstrated yet and confounding factors, such as binding by Kuppfer cells in the liver, have still to be addressed.

Ox-LDL binds to the ox-LDL receptor LOX-1 and induces apoptosis, expression of adhesion molecules, and release of MMPs [1, 31]. Injection of paramagnetic liposomes conjugated with anti-LOX-1 antibodies in LDL-R-/- mice induced specific MRI-signal enhancement in plaques in the aorta. Enhancement was neither observed in double knockout LDL-R-/- LOX-1-/- mice nor in LDL-R-/- mice injected with nonspecific IgG-conjugated liposomes [31]. Histological analyses revealed that most LOX-1 co-localized with macrophages, apoptotic cells, and MMP-9 and only a small proportion with proliferated smooth muscle cells.

Distinct disadvantages of the targeted approach with antibodies are the difficulty in handling and preparation, costs of antibodies, immunogenicity, and possible steric hindrance by PEG reducing the specific interactions. An alternative approach is the use of specific targeting moieties of non-antibody origin. An example is the use of $\mathrm{Gd}-$ containing liposomes with phosphatidylserine (PS) incorporated in the lipid membrane. PS is expressed on apoptotic cells in the plaque and is specifically recognized by macrophages. Using that same mechanism, PS-containing liposomes are specifically phagocytosed by macrophages in the plaque. In vitro PS-containing liposomes showed specificity for RAW cells (murine macrophages), and significant enhancement of plaques in the aortic wall of apoE-/mice was observed in vivo up to $4 \mathrm{~h}$ after injection [32].

In another study, plaque macrophages were targeted using a potent synthetic agonist (HU-308) of the peripheral cannabinoid receptor (CB2-R) [33]. CB2-R is expressed by T-lymphocytes and macrophages in advanced murine and human atherosclerotic plaque and is believed to dampen the inflammatory process in advanced plaques [34]. HU-308, which has a 7-carbon tail and a hydrophilic head group, was mixed with Gd-containing and PEG-ylated lipids to form MRI-visible micelles [33]. In vivo plaque enhancement in the aortas of double knockout apoE-/- eNOS-/mice was observed from 36 to $48 \mathrm{~h}$ after injection of the CB2-R-targeted micelles using black-blood $\mathrm{T}_{1}$-weighted inversion recovery fast spin echo MRI [35].

HDL-like nanoparticles functionalized with an apoEderived lipopeptide were used to target macrophages in the atherosclerotic lesions in the aorta of apoE-/- mice [36]. Various versions of HDL-mimicking nanoparticles have been designed for plaque imaging [37]. An attractive property of such particles is that HDL is of endogenous nature, small, and plays a key role in plaque etiology. Among others, HDL enables reverse cholesterol transport from the plaque, has anti-atherothrombotic properties, and reduces oxidation and plaque inflammation. These MRIvisible nanoparticles might be useful to study the role of HDL in plaque progression [38].

Iron oxides with a lipid coating and conjugated with hyaluronic acid (HA), which targets the CD44 receptor on activated macrophages, were employed to study plaque inflammation as well [39]. Cellular uptake of these CD44targeted iron oxides was demonstrated in vitro for activated THP-1 macrophages. Prussian blue stainings for iron revealed that the nanoparticles were only transiently present in the cells, which is beneficial in view of possible toxicity issues. In vivo application of these macrophage-targeted iron oxides has still to be demonstrated.

Apart from direct targeting of macrophages, one can also address the presence of extracellular enzymes or proteins in the plaque associated with inflammation. Plaque rupture has been attributed to increased activity of matrix metalloproteinases (MMPs) produced by SMCs and macrophages [40]. In vivo MR imaging of plaque MMP expression has been demonstrated using a small peptide with affinity for MMPs covalently bound to Gd-DOTA [41]. Specific inhibitors of MMP-2 and MMP-9 may be used for improved targeting of MMPs in the plaque. A protein that has been linked to adverse cardiovascular events in patients after carotid endarterectomy surgery is neutrophil gelatinase-associated lipocalin-2 (NGAL) [42]. First discovered in human neutrophils, NGAL is produced and released particularly by macrophages. It is able to form a stable, biologically active 
complex with MMP-9, preventing its degradation and thereby prolonging MMP-9 activity. Additionally, it mediates inflammatory activity through binding to formyl-methionyl-leucyl-phenylalanine (fMLP; a chemotactic peptide), leukotriene B4, and platelet-activating factor. As summarized in Fig. 1, the mouse homologue of NGAL, 24p3, was successfully imaged with $24 \mathrm{p} 3$-targeted micelles in severe atherosclerotic plaques of the double knockout apoE-/eNOS-/- mouse [42]. Histology proved that micelles colocalized with $24 \mathrm{p} 3$ and partially with macrophages.

On the one hand, targeting of extracellular-located molecules such as MMPs and NGAL has the advantage that the target is readily available. On the other hand, it may carry the drawback that the contrast agent is easily washed out from the plaque into the blood. Nevertheless, imaging of excreted proteins could provide important insights in the inflammatory state of the plaque. For example, peroxidasebased hematologic profiling has been shown to predict adverse cardiac events during a follow-up of 1 year [43]. In order to prevent washout of the agent, Ronald and coworkers [44••] have designed an activatable (smart) Gdcontaining probe for imaging of myeloperoxidase, an enzyme released by macrophages that is responsible for oxidation of LDL via the formation of reactive oxygen species. Activation of the probe by myeloperoxidase leads to polymerization, increasing its size and preventing washout from the target region-hence providing an indirect readout of enzyme activity. As summarized in Fig. 2, with this probe increased myeloperoxidase activity was imaged in plaques in the aorta of rabbits [44••].

\section{Apoptotic Cells}

One of the characteristics of apoptotic cells is that PS, which is only found in the inner leaflet of the viable cell membrane, becomes expressed on the outer leaflet of the cell. The expression of PS can be used for MR imaging of apoptosis by using the protein annexin A5 as a targeting ligand. Annexin A5 binds specifically to PS in the presence of calcium. Gd-containing micelles, conjugated with annexin A5, were employed to study apoptosis in atherosclerotic plaques in the aorta of apoE-/- mice. Twenty-four hours after intravenous injection, enhancement of the plaque was observed on $\mathrm{T}_{1}$-weighted $\mathrm{MRI}$, which was slightly, but significantly, higher than for control nonspecific micelles [45]. In another study, a PS-specific peptide, which was identified by phage display, was conjugated to Gd-DTPA. Thirty minutes after intravenous injection, a specific enhancement of plaques in the aorta of apoE-/mice was observed [46]. The use of small peptides has certain advantages above proteins or antibodies in terms of reduced immunogenicity, as well as a cheaper and easier production process.
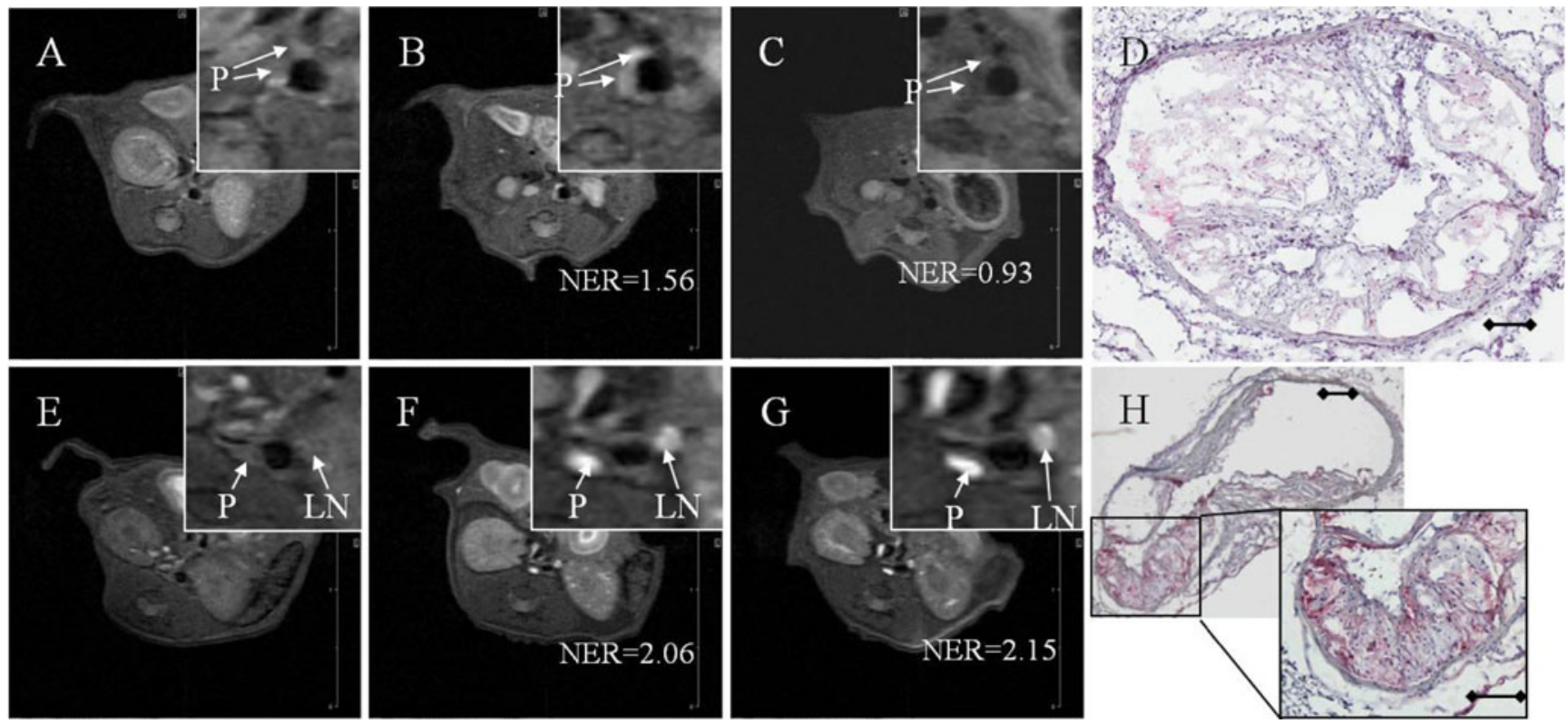

Fig. 1 Magnetic resonance (MR) images and histological stainings of murine atherosclerotic plaque after intravenous injection of NGAL/ 24p3-targeted micelles. Pre-injection and post-injection $(0,24$, and $72 \mathrm{~h}) \mathrm{MR}$ images of aortic wall in apoE $\mathrm{E}^{-/-} / \mathrm{eNOS}^{-/-}$mice injected with control $(\mathbf{a}-\mathbf{c})$ and NGAL/24p3-targeted $(\mathbf{e}-\mathbf{g})$ micelles. Histology of the mouse aorta at $72 \mathrm{~h}$ after injection revealed no staining of micelles for injections with control micelles (d) and extensive red staining for injections with NGAL/24p3-targeted micelles (h). The post-injection MR images show plaque enhancement and also enhancement of a lymph node, which was removed during harvesting of the aorta. H: scale bar $=100 \mu \mathrm{m}$ (original magnification: $100 \times$ ). LN, lymph node; $\mathrm{P}$, plaque 

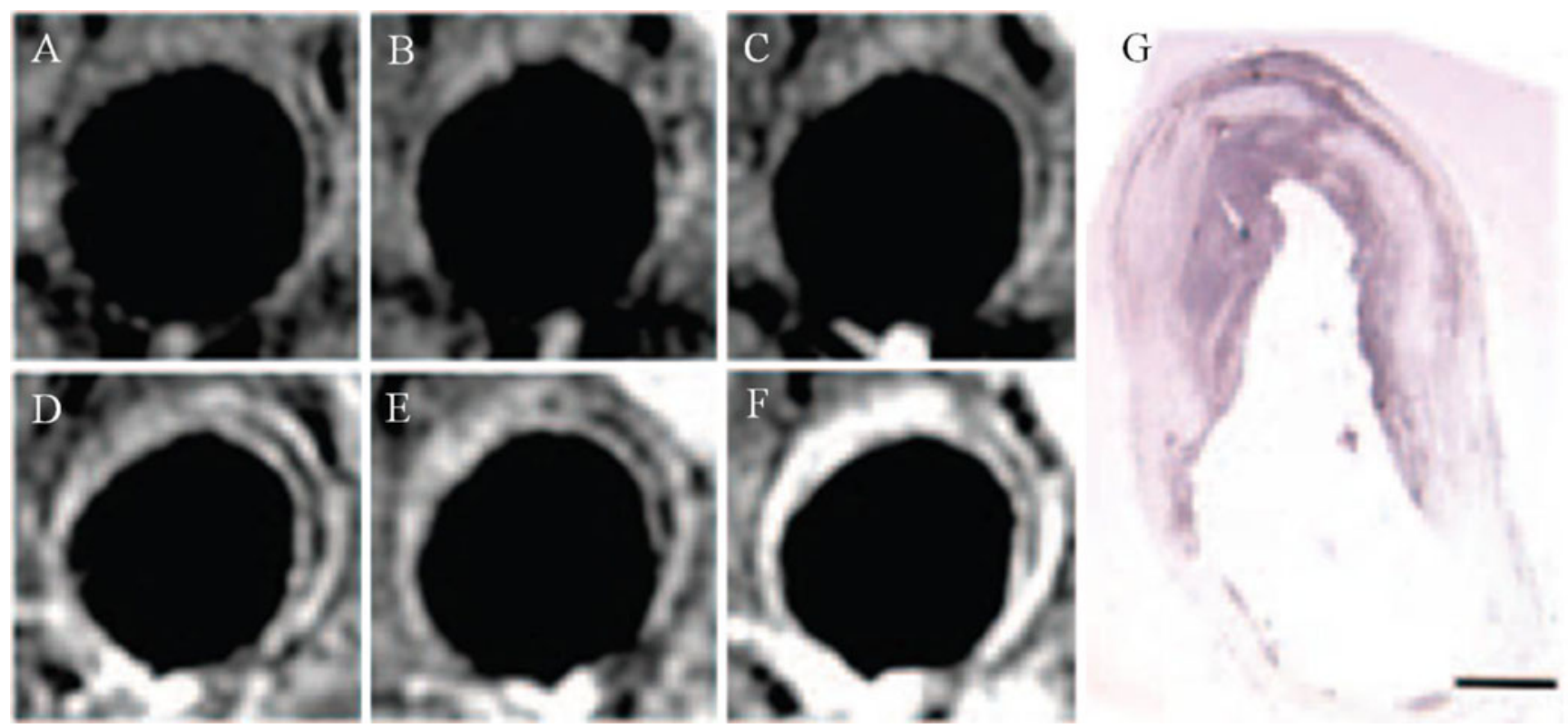
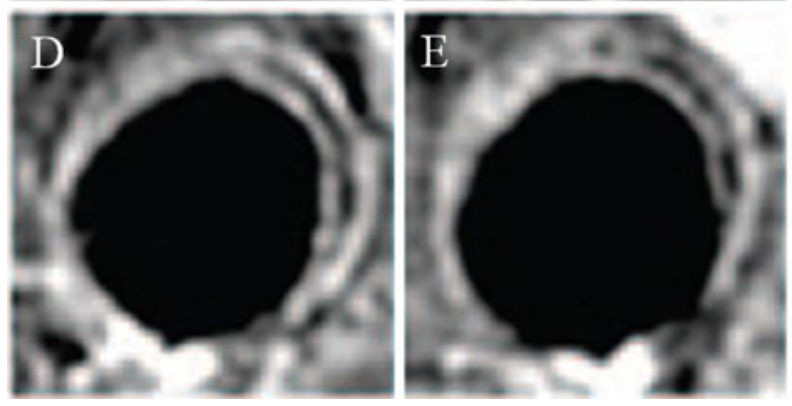

Fig. 2 Magnetic resonance (MR) images and histological staining of rabbit atherosclerotic plaque after injection of an activatable MPO sensor. Imaging of aortas of cholesterol-fed rabbits before and $2 \mathrm{~h}$ after injection of DTPA-Gd (a, d), before and $2 \mathrm{~h}$ after injection of bistyr-DTPA-Gd (b, e), which is a non-activatable analog of MPO-Gd,

\section{Activated Platelets and Thrombus}

Activated platelets play a crucial role in the formation of micro-thrombi, which eventually may accumulate in an acute cardiovascular event. Therefore, early recognition of frequently occurring minor atherothrombosis has important prognostic value. Ultra-small particles of iron oxide have been conjugated with cyclic arginine-glycineaspartic acid (RGD) peptides specific for the $\alpha \mathrm{II} \beta 3$ integrin among others, which is expressed on activated platelets [47]. Unfortunately, the resolution, which was achieved with RGD-USPIO-enhanced MRI, was too low to detect thrombus in vivo. Recently, single-chain antibodies ( $\mathrm{scFv}$ ) have been successfully conjugated to iron nanoparticles using the Staphylococcus aureus sortase A enzyme for molecular imaging in cardiovascular disease. Binding of scFv-conjugated nanoparticles to activated platelets was demonstrated in vivo in mouse carotid arteries [48]. Another single-chain antibody aimed to target ligand-induced binding sites on activated glycoprotein IIb/IIIa was conjugated to microparticles of iron oxide (diameter $1 \mu \mathrm{m}$ ) [49]. Ferric chloride-induced thrombi were imaged repeatedly in mice after injection of this contrast agent with a significant decrease of signal intensity at $36 \mathrm{~min}$ after injection [49]. After thrombolysis the signal increased again, indicating that this approach enables the evaluation of the efficacy of thrombolytic therapy. and before and $2 \mathrm{~h}$ after injection of MPO-Gd (c, f). Histology of the rabbit aorta (g) after injection of MPO-Gd revealed focal MPO presence in the plaque and confirmed the MR findings. (From Ronald et al. [44••]; with permission)

Various products of the coagulation cascade may serve as targets for molecular MRI. Fibrin-targeted small-molecule peptides conjugated to Gd-DTPA have been applied successfully in patients to image thrombus [50••]. Fibrin-specific Gdperfluorocarbon nanoparticles were also applied for MR imaging of thrombus [51]. The perfluorocarbon nanoparticles can be used for $\mathrm{T}_{1}$-weighted proton MRI as well as for ${ }^{19} \mathrm{~F}$ hot-spot MR imaging. Finally, also contrast agents based on the chemical exchange saturation transfer (CEST) mechanism [52] have been employed for imaging of fibrin. Proof-of-concept CEST imaging of fibrin was provided in vitro for fibrin clots, using a fibrin-specific perfluorocarbon nanoparticle containing a paraCEST compound [53•]. The CEST agents are potentially attractive for in vivo use, because contrast can be switched on and off by selective RF pulse saturation, avoiding the need for pre-injection and post-injection imaging which is for clinical application and not attractive from a logistical point of view.

\section{Limitations}

As described above, MRI is capable of providing detailed cellular and molecular imaging information, which combined with the wealth of anatomical and functional MRI readouts, makes MR imaging of atherosclerosis a very powerful asset to study plaque vulnerability, progression, and to evaluate effects of treatment. A potential limitation 
of MRI, however, is the limited sensitivity of the technique in the detection of low concentrations of targeted contrast agent. Although single-cell detection with the use of iron oxides has been reported, in general, much higher doses are needed as compared to, for example, the nuclear imaging techniques [2]. This complicates rapid approval of MR contrast agents and its carriers for human application by the FDA (USA) and EMEA (Europe), because of the concerns about retention in the body and toxicity. Issues are, amongst others, the unknown metabolic fate of the carrier material in the body, transmetallation of $\mathrm{Gd}^{3+}$ with native metal-ions in the body [4], and worries about Gd-induced nephrogenic systemic fibrosis [6].

The use of frequency-selective paraCEST agents or perfluorocarbon nanoparticles permits imaging of various target species simultaneously. Although in vitro proof-of-principle has been provided, in vivo application has been limited so far. Problems for paraCEST agents are related to low sensitivity and susceptibility artifacts, whereas ${ }^{19} \mathrm{~F}$ imaging also suffers from relatively low sensitivity. Concerning clinical translation, the iron oxides are most promising, since they enable the most sensitive detection while toxicity is low. The larger iron oxides provide sensitive hypointense contrast on $\mathrm{T}_{2}$-weighted images, whereas small iron oxides $(\sim 5 \mathrm{~nm})$ can also be used for $\mathrm{T}_{1}$-weighted imaging with hyperintense contrast $[54 \bullet]$.

\section{Conclusions}

As reviewed in this paper, in recent years a number of MRI strategies to image inflammation in atherosclerotic plaques were successfully developed. Proof-of-concept clinical application was already established for imaging of macrophages using non-targeted iron oxides. Future studies will have to clarify whether the targeted contrast agents will have diagnostic or prognostic added value in the evaluation of atherosclerotic plaque inflammation in humans. Promising new developments include activatable contrast agents, which are able to report on enzyme activity, as well as the use of MRI-visible endogenous nanoparticles, such as HDL and LDL, for imaging of atherosclerosis and plaque inflammation.

Disclosure No potential conflicts of interest relevant to this article were reported.

Open Access This article is distributed under the terms of the Creative Commons Attribution Noncommercial License which permits any noncommercial use, distribution, and reproduction in any medium, provided the original author(s) and source are credited.

\section{References}

Papers of particular interest, published recently, have been highlighted as:

- Of importance

•. Of major importance

1. Ross R. Atherosclerosis is an inflammatory disease. Am Heart J. 1999;138:S419-20.

2. Leuschner F, Nahrendorf M. Molecular imaging of coronary atherosclerosis and myocardial infarction: considerations for the bench and perspectives for the clinic. Circ Res. 2011;108:593-606.

3. Briley-Saebo KC, Shaw PX, Mulder WJ, et al. Targeted molecular probes for imaging atherosclerotic lesions with magnetic resonance using antibodies that recognize oxidation-specific epitopes. Circulation. 2008;117:3206-15.

4. Hak S, Sanders HM, Agrawal P, et al. A high relaxivity Gd(III) DOTA-DSPE-based liposomal contrast agent for magnetic resonance imaging. Eur J Pharm Biopharm. 2009;72:397-404.

5. Ide M, Kuwamura M, Kotani T, et al. Effects of gadolinium chloride $(\mathrm{GdCl}(3))$ on the appearance of macrophage populations and fibrogenesis in thioacetamide-induced rat hepatic lesions. J Comp Pathol. 2005;133:92-102.

6. Pedersen M. Safety update on the possible causal relationship between gadolinium-containing MRI agents and nephrogenic systemic fibrosis. J Magn Reson Imaging. 2007;25:881-3.

7. Briley-Saebo KC, Cho YS, Shaw PX, et al. Targeted iron oxide particles for in vivo magnetic resonance detection of atherosclerotic lesions with antibodies directed to oxidation-specific epitopes. J Am Coll Cardiol. 2011;57:337-47.

8. McAteer MA, Akhtar AM, von Zur MC, et al. An approach to molecular imaging of atherosclerosis, thrombosis, and vascular inflammation using microparticles of iron oxide. Atherosclerosis. 2010;209:18-27.

9. Akhtar AM, Schneider JE, Chapman SJ, et al. In vivo quantification of VCAM-1 expression in renal ischemia reperfusion injury using non-invasive magnetic resonance molecular imaging. PLoS One. 2010;5:e12800.

10. Kelly KA, Allport JR, Tsourkas A, et al. Detection of vascular adhesion molecule-1 expression using a novel multimodal nanoparticle. Circ Res. 2005;96:327-36.

11. Nahrendorf M, Jaffer FA, Kelly KA, et al. Noninvasive vascular cell adhesion molecule-1 imaging identifies inflammatory activation of cells in atherosclerosis. Circulation. 2006;114:1504-11.

12. Alsaid H, De SG, Bourdillon MC, et al. Biomimetic MRI contrast agent for imaging of inflammation in atherosclerotic plaque of ApoE-/- mice: a pilot study. Invest Radiol. 2009;44:151-8.

13. Sluimer JC, Daemen MJ. Novel concepts in atherogenesis: angiogenesis and hypoxia in atherosclerosis. J Pathol. 2009;218:729.

14. Dunmore BJ, McCarthy MJ, Naylor AR, et al. Carotid plaque instability and ischemic symptoms are linked to immaturity of microvessels within plaques. J Vasc Surg. 2007;45:155-9.

15. Takaya N, Yuan C, Chu B, et al. Presence of intraplaque hemorrhage stimulates progression of carotid atherosclerotic plaques: a high-resolution magnetic resonance imaging study. Circulation. 2005;111:2768-75.

16. Yuan C, Kerwin WS, Ferguson MS, et al. Contrast-enhanced high resolution MRI for atherosclerotic carotid artery tissue characterization. J Magn Reson Imaging. 2002;15:62-7.

17. Calcagno C, Mani V, Ramachandran S, et al. Dynamic contrast enhanced (DCE) magnetic resonance imaging (MRI) of atherosclerotic plaque angiogenesis. Angiogenesis. 2010;13:8799. 
18. Barkhausen J, Ebert W, Heyer C, et al. Detection of atherosclerotic plaque with Gadofluorine-enhanced magnetic resonance imaging. Circulation. 2003;108:605-9.

19. Meding J, Urich M, Licha K, et al. Magnetic resonance imaging of atherosclerosis by targeting extracellular matrix deposition with gadofluorine M. Contrast Media Mol Imaging. 2007;2:120-9.

20. Sirol M, Moreno PR, Purushothaman KR, et al. Increased neovascularization in advanced lipid-rich atherosclerotic lesions detected by gadofluorine-M-enhanced MRI: implications for plaque vulnerability. Circ Cardiovasc Imaging. 2009;2:391-6.

21. Cai K, Caruthers SD, Huang W, et al. MR molecular imaging of aortic angiogenesis. JACC Cardiovasc Imaging. 2010;3:824-32.

22. Winter PM, Caruthers SD, Allen JS, et al. Molecular imaging of angiogenic therapy in peripheral vascular disease with alphanubeta3-integrin-targeted nanoparticles. Magn Reson Med. 2010;64:369-76.

23. Schmitz SA. Iron-oxide-enhanced MR imaging of inflammatory atherosclerotic lesions: overview of experimental and initial clinical results. Rofo. 2003;175:469-76.

24. Schmitz SA, Taupitz M, Wagner S, et al. Magnetic resonance imaging of atherosclerotic plaques using superparamagnetic iron oxide particles. J Magn Reson Imaging. 2001;14:355-61.

25. Te Boekhorst BC, Bovens SM, Nederhoff MG, et al. Negative MR contrast caused by USPIO uptake in lymph nodes may lead to false positive observations with in vivo visualization of murine atherosclerotic plaque. Atherosclerosis. 2010;210:122-9.

26. Kooi ME, Cappendijk VC, Cleutjens KB, et al. Accumulation of ultrasmall superparamagnetic particles of iron oxide in human atherosclerotic plaques can be detected by in vivo magnetic resonance imaging. Circulation. 2003;107:2453-8.

27. Tang T, Howarth SP, Miller SR, et al. Assessment of inflammatory burden contralateral to the symptomatic carotid stenosis using high-resolution ultrasmall, superparamagnetic iron oxideenhanced MRI. Stroke. 2006;37:2266-70.

28. Tang TY, Howarth SP, Miller SR, et al. The ATHEROMA (atorvastatin therapy: effects on reduction of macrophage activity) study. Evaluation using ultrasmall superparamagnetic iron oxideenhanced magnetic resonance imaging in carotid disease. $\mathrm{J}$ Am Coll Cardiol. 2009;53:2039-50.

29. Amirbekian V, Lipinski MJ, Briley-Saebo KC, et al. Detecting and assessing macrophages in vivo to evaluate atherosclerosis noninvasively using molecular MRI. Proc Natl Acad Sci U S A. 2007;104:961-6.

30. Lipinski MJ, Frias JC, Amirbekian V, et al. Macrophage-specific lipid-based nanoparticles improve cardiac magnetic resonance detection and characterization of human atherosclerosis. JACC Cardiovasc Imaging. 2009;2:637-47.

31. Li D, Patel AR, Klibanov AL, et al. Molecular imaging of atherosclerotic plaques targeted to oxidized LDL receptor LOX-1 by SPECT/CT and magnetic resonance. Circ Cardiovasc Imaging. 2010;3:464-72.

32. Maiseyeu A, Mihai G, Kampfrath T, et al. Gadolinium-containing phosphatidylserine liposomes for molecular imaging of atherosclerosis. J Lipid Res. 2009;50:2157-63.

33. Te Boekhorst BC, Bovens SM, Rodrigues-Feo J, et al. Characterization and in vitro and in vivo testing of CB2-receptor- and NGALtargeted paramagnetic micelles for molecular MRI of vulnerable atherosclerotic plaque. Mol Imaging Biol. 2010;12:635-51.

34. Steffens S, Veillard NR, Arnaud C, et al. Low dose oral cannabinoid therapy reduces progression of atherosclerosis in mice. Nature. 2005;434:782-6.

35. Te Boekhorst BC, Bovens SM, van de Kolk CW, et al. The time window of MRI of murine atherosclerotic plaques after administration of CB2 receptor targeted micelles: inter-scan variability and relation between plaque signal intensity increase and gadolinium content of inversion recovery prepared versus nonprepared fast spin echo. NMR Biomed. 2010;23:939-51.

36. Chen W, Vucic E, Leupold E, et al. Incorporation of an apoEderived lipopeptide in high-density lipoprotein MRI contrast agents for enhanced imaging of macrophages in atherosclerosis. Contrast Media Mol Imaging. 2008;3:233-42.

37. Cormode DP, Briley-Saebo KC, Mulder WJ, et al. An ApoA-I mimetic peptide high-density-lipoprotein-based MRI contrast agent for atherosclerotic plaque composition detection. Small. 2008;4:1437-44.

38. Skajaa T, Cormode DP, Falk E, et al. High-density lipoproteinbased contrast agents for multimodal imaging of atherosclerosis. Arterioscler Thromb Vasc Biol. 2010;30:169-76.

39. Kamat M, El-Boubbou K, Zhu DC, et al. Hyaluronic acid immobilized magnetic nanoparticles for active targeting and imaging of macrophages. Bioconjug Chem. 2010;21:2128-35.

40. Schafers M, Schober O, Hermann S. Matrix-metalloproteinases as imaging targets for inflammatory activity in atherosclerotic plaques. J Nucl Med. 2010;51:663-6.

41. Lancelot E, Amirbekian V, Brigger I, et al. Evaluation of matrix metalloproteinases in atherosclerosis using a novel noninvasive imaging approach. Arterioscler Thromb Vasc Biol. 2008;28:425-32.

42. Te Boekhorst BC, Bovens SM, Hellings WE, et al. Molecular MRI of murine atherosclerotic plaque targeting NGAL: a protein associated with unstable human plaque characteristics. Cardiovasc Res. 2011;89:680-8.

43. Brennan ML, Reddy A, Tang WH, et al. Comprehensive peroxidase-based hematologic profiling for the prediction of 1-year myocardial infarction and death. Circulation. 2010; 122:70-9.

44. •• Ronald JA, Chen JW, Chen Y, et al. Enzyme-sensitive magnetic resonance imaging targeting myeloperoxidase identifies active inflammation in experimental rabbit atherosclerotic plaques. Circulation. 2009;120:592-9. In vivo potential of activatable contrast agents for visualization of enzyme activity in plaques with MRI.

45. van Tilborg GA, Vucic E, Strijkers GJ, et al. Annexin A5functionalized bimodal nanoparticles for MRI and fluorescence imaging of atherosclerotic plaques. Bioconjug Chem. 2010;21:1794-803

46. Burtea C, Laurent S, Lancelot E, et al. Peptidic targeting of phosphatidylserine for the MRI detection of apoptosis in atherosclerotic plaques. Mol Pharm. 2009;6:1903-19.

47. Johansson LO, Bjornerud A, Ahlstrom HK, et al. A targeted contrast agent for magnetic resonance imaging of thrombus: implications of spatial resolution. J Magn Reson Imaging. 2001;13:615-8.

48. Ta HT, Prabhu S, Leitner E, et al. Enzymatic single-chain antibody tagging: a universal approach to targeted molecular imaging and cell homing in cardiovascular disease. Circ Res. 2011, Epub ahead of print.

49. Von zur Muhlen C, Von Elverfeldt D, Moeller JA, et al. Magnetic resonance imaging contrast agent targeted toward activated platelets allows in vivo detection of thrombosis and monitoring of thrombolysis. Circulation. 2008;118:258-67.

50. •- Spuentrup E, Botnar RM, Wiethoff AJ, et al. MR imaging of thrombi using EP-2104R, a fibrin-specific contrast agent: initial results in patients. Eur Radiol. 2008;18:1995-2005. In vivo clinical applicability of small gadolinium-based fibrin-targeted contrast agent.

51. Morawski AM, Winter PM, Yu X, et al. Quantitative "magnetic resonance immunohistochemistry" with ligand-targeted (19)F nanoparticles. Magn Reson Med. 2004;52:1255-62.

52. Ward KM, Aletras AH, Balaban RS. A new class of contrast agents for MRI based on proton chemical exchange dependent saturation transfer (CEST). J Magn Reson. 2000;143:79-87. 
53. - Winter PM, Cai K, Chen J, et al. Targeted PARACEST nanoparticle contrast agent for the detection of fibrin. Magn Reson Med. 2006;56:1384-8. An example of fibrin-targeting with a paraCEST contrast agent with possible transfer to the clinic.

54. - Taboada E, Rodriguez E, Roig A, et al. Relaxometric and magnetic characterization of ultrasmall iron oxide nanoparticles with high magnetization. Evaluation as potential T1 magnetic resonance imaging contrast agents for molecular imaging. Langmuir. 2007;23:4583-8. Description of the T1 contrast properties of iron oxide-based nanoparticles, which have less toxicity issues when compared to gadolinium-based nanoparticles. Positive contrast caused by shortened T1 is not masked by susceptibility artifacts and area of contrast uptake will not be overestimated as is the case with $T 2 *$ contrast. 